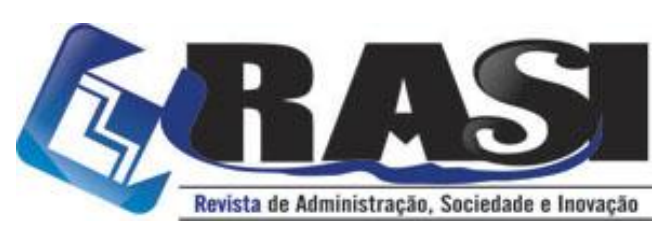

http://www.rasi.vr.uff.br

RASI, Volta Redonda/RJ, v. 7, n. 1, pp. 104-123, Jan./Abr. 2021

\title{
Subvenção à Inovação no Setor Farmacêutico Brasileiro: Uma Análise a partir do Nível de Incerteza
}

Luiza Pinheiro (UFMG) - luizapasilva@gmail.com

Márcia Siqueira Rapini (UFMG) - msrapini@ cedeplar.ufmg.br

Julia Paranhos (UFRJ) - juliaparanhos@ie.ufrj.br

\begin{abstract}
Resumo:
$\mathrm{O}$ artigo analisa de forma pioneira o grau de incerteza dos projetos do setor farmacêutico que receberam recursos de subvenção econômica da FINEP entre 2007 e 2014. Foram analisados 87 projetos de 60 empresas contemplados em 8 editais de subvenção. Os dados indicam um aumento do valor médio por projeto, bem como oscilações ao longo do período no aporte de recursos para projetos deste setor, ainda que seja um setor sistematicamente contemplado pelas recentes políticas industriais. A partir da taxonomia de incerteza e tipos de inovação de Freeman e Soete, 52 projetos do setor farmacêutico foram classificados, caracterizando-se como um estudo multicaso. Esta análise permitiu inferir que, na média, os projetos apoiados envolvem incerteza moderada, apontando para a importância de políticas industriais sistêmicas que incentivem que as empresas brasileiras farmacêuticas avancem no desenvolvimento de atividades de inovação com maior grau de incerteza.

Palavras-Chaves: Subvenção Econômica; FINEP; Setor Farmacêutico; Incerteza.
\end{abstract}

\section{Innovation Grant in the Brazilian Pharmaceutical Sector: An Analysis from the Level of Uncertainty}

\begin{abstract}
:
The article analyzes in a pioneering way the degree of uncertainty of the pharmaceutical sector projects that received grant resources from FINEP between 2007 and 2014. We analyzed 87 projects from 60 companies included in 8 grant announcements. The data indicates an increase in the average value per project, as well as fluctuations over the period in the provision of resources, although it is a sector systematically contemplated by the recent industrial policies. From the taxonomy of uncertainty and types of innovation by Freeman and Soete, 52 projects in the pharmaceutical sector were classified. This analysis allowed to infer that, on average, the supported projects involve moderate uncertainty, pointing to the importance of systemic industrial policies that encourage Brazilian pharmaceutical companies to advance in development innovation activities with a greater degree of uncertainty.
\end{abstract}

Keywords: Public Grants; FINEP; Pharmaceutical Sector; Uncertainty.

\begin{tabular}{c} 
R. Desembargador Ellis Hermydio Figueira, 783, Bloco A, sl. 218, Aterrado. \\
$27213-415$ - Volta Redonda, RJ - Brasil \\
www.uff.br \\
$\underline{\text { Federal }}$ \\
\hline $\begin{array}{c}\text { Copyright (c) 2020 RASI. Todos os direitos, até mesmo de tradução, são reservados. É } \\
\text { permitido citar parte de artigos sem autorização prévia, desde que seja identificada a fonte. }\end{array}$ \\
\hline
\end{tabular}




\section{Subvenção à Inovação no Setor Farmacêutico Brasileiro: Uma Análise a partir do Nível de Incerteza}

\section{Introdução}

Em vista das características dos investimentos em inovação requererem elevados montantes, grande incerteza e longo prazo de retorno há a necessidade de se criar instrumentos e mecanismos de financiamento diferenciados, por parte das empresas, dos mercados financeiros e pela ação explícita dos governos (Frenkel, 1993). Desta forma, as fontes de financiamento para os investimentos em inovação podem ser públicas ou privadas, na maioria das vezes, sendo um combinado de ambas.

O financiamento público, em muitos países, é de longo prazo reunindo: (1) programas para incentivar as empresas a realizarem Pesquisa e Desenvolvimento (P\&D) através de empréstimos, recursos não-reembolsáveis e incentivos fiscais; (2) recursos para o financiamento de P\&D nas empresas através de encomendas tecnológicas; (3) suporte para o estabelecimento de infraestrutura de P\&D para o desenvolvimento industrial (Chesnais, \& Sauviat, 2005).

Dentre os incentivos não fiscais de apoio à inovação nas empresas há duas modalidades associadas à concessão direta de recursos para as empresas, por meio do aporte de recursos ou da participação nos custos (Longo, et al., 2002). O aporte de recursos pode se dar através de empréstimos com taxas reduzidas de juros, participação acionária e capital de risco. A participação nos custos pode ser parcial direta, parcial indireta ou total através de encomenda/compra de desenvolvimento de produtos/processos. Estes instrumentos requerem escolhas prévias do governo que no geral estão associadas a projetos e/ou áreas especiais. Portanto, esta forma de funding requer mais informação sobre os potenciais benefícios sociais e privados dos gastos pretendidos, o que pode produzir melhores resultados, do que simplesmente deixar para as empresas a escolha de seus projetos.

Por sua vez, os subsídios diretos às empresas são ranqueados pelo governo de acordo com gap percebido existente entre os retornos privado e social. Seus resultados não são tão claros, mas tendem a ser mais efetivos nas pequenas empresas e start-ups que possuem poucas obrigações fiscais (Hall, 2002). Além do mais, os recursos para financiamento direto, no mínimo, devem ser baseados em comprometimentos de longo prazo de forma a reduzir a vulnerabilidade e restrições ad hoc impostas por flutuações de curto prazo no orçamento público (Penedo, 2008).

No Brasil, os subsídios para as empresas na modalidade de subvenção econômica são relativamente recentes, tendo sido instituídos pela Lei 10.332/2001, ainda de forma restrita, e ampliados pela Lei 10.973/2004 (Lei de Inovação), e seu Decreto Regulamentar 5.563/2005. Sendo assim, a operacionalização destes recursos não-reembolsáveis se iniciou de forma efetiva em 2006 pela Financiadora de Estudos e Projetos (FINEP) por meio de chamadas públicas do Programa de Subvenção Econômica. No início dos anos 2000, a agenda de política industrial e tecnológica volta à pauta do governo por meio da implementação da Política Industrial, Tecnológica e de Comércio Exterior (PITCE) e tem continuidade, em 2008, com a Política de Desenvolvimento Produtivo (PDP) e, em 2011, com o Plano Brasil 
Maior (PBM). Em todos os casos o setor farmacêutico ${ }^{1}$ foi definido como estratégico para o desenvolvimento do país. Neste contexto, instrumentos de apoio, financiamento e estímulo à inovação são estabelecidos e/ou direcionados para o setor farmacêutico, entre eles o Programa de subvenção econômica da FINEP (Hasenclever, et al., 2016).Tais ações foram relevantes para o desenvolvimento do setor em termos nacionais e internacionais. Em cinco anos, o Brasil passou de $10^{\circ}$ para o $8^{\circ}$ mercado mundial e o mercado interno cresceu cerca de $50 \%$ em valor ( $\mathrm{R} \$ 33$ bilhões para $\mathrm{R} \$ 50$ bilhões), com forte ampliação da participação das empresas nacionais, que eram seis entre as 10 maiores empresas do mercado brasileiro em 2016 (Interfarma, 2017).

No entanto, apesar da grande importância dada ao desenvolvimento da inovação como um todo e, em especial, ao setor farmacêutico, não foi estabelecida uma metodologia sistemática de acompanhamento, avaliação e revisão destes instrumentos. Dada a continuidade do setor farmacêutico como alvo das políticas e instrumentos de estímulo à inovação, neste trabalho, propõe-se um estudo multicaso dos projetos do setor farmacêutico apoiados pela subvenção econômica da FINEP. O foco da análise levará em conta o nível da incerteza, segundo classificação de Freeman e Soete (2008), presente nos projetos apoiados. Não se pretende aqui ser exaustivo na análise do setor, dado que as características setoriais nacionais e internacionais, atuais e históricas, em relação às empresas, às instituições científicas e tecnológicas, ao marco regulatório, institucional e político também podem afetar (e provavelmente afetam) o nível de incerteza dos projetos submetidos e apoiados. No entanto, busca-se levantar elementos para o debate sobre os instrumentos de apoio à inovação para o setor farmacêutico no Brasil.

Sendo assim, o objetivo neste artigo é, de forma pioneira, analisar o Programa de subvenção econômica a partir do nível de incerteza dos projetos do setor farmacêutico apoiados pela FINEP entre 2007 e 2014. O artigo está dividido em quatro seções, ademais desta Introdução e da Conclusão. A segunda seção apresenta especificidades do processo de inovação e de seu financiamento no setor farmacêutico. A terceira seção apresenta de forma breve a Subvenção Econômica na FINEP e a evolução dos recursos aportados e do número de projetos apoiados. A quarta seção apresenta a metodologia construída para a classificação dos projetos do setor farmacêutico de acordo com o nível de incerteza. A quinta seção apresenta os dados gerais dos projetos apoiados do setor farmacêutico, bem como o resultado da classificação de acordo com o nível de incerteza. A última seção conclui o trabalho, apresentando reflexões para a proposição de políticas de inovação.

\section{Financiamento à Inovação, Incerteza e o Setor Farmacêutico no Brasil}

Conforme apresenta Fagerberg (2005), desde seus primeiros trabalhos Schumpeter (1911) ressaltou a incerteza inerente em todos os projetos inovativos, e trabalhos futuros, como Nelson e Winter (1982) e Nonaka e Takeuchi(1995), corroboraram a sua afirmação. O processo de geração de inovação incorre em incertezas ${ }^{2}$ desde as atividades de pesquisa e, de desenvolvimento tecnológico, até a introdução no mercado. As empresas buscam se organizar

\footnotetext{
${ }^{1} \mathrm{Na}$ PITCE, o setor de fármacos e medicamentos foi identificado como um dos quatro setores estratégicos. Na PDP, o Complexo Industrial da Saúde, que inclui o setor farmacêutico, está listado entre os 25 setores definidos como estratégicos; e segue assim no PBM.

${ }^{2}$ Grande parte dos economistas diferencia as incertezas calculáveis, ou riscos, das incertezas não calculáveis ou a incerteza de fato, partindo do trabalho do Knight (1965).
} 
e implementar estratégias para reduzir esta incerteza, mas ela estará sempre intrínseca no processo. Dentre outras coisas, pode-se mencionar que os investimentos em atividades inovativas são caracterizados por: (1) produção de ativos intangíveis de difícil mensuração (Nakamura, 1999); (2) indivisibilidade do conhecimento; (3) dificuldade de apropriação privada dos benefícios ${ }^{3}$; (4) elevada assimetria de informação entre o ofertante de crédito e o demandante (Christensen, 1992). Algumas tentativas de inovação alcançam sucesso, refletindo em novos produtos, serviços, processos ou até mesmo empresas. Outras não são bem-sucedidas, por razões técnicas, comerciais ou pela incapacidade de prever os rumos do processo de concorrência. De acordo com Freeman e Soete (2008) "o que pode ser reconhecido ex-post nem sempre pode ser controlado neste ou iniciado ex-ante", tendo em vista que muitas das variáveis envolvidas não são fáceis de manipular. Em suma, os resultados não podem ser previstos perfeitamente a partir dos insumos.

Dosi (1988) acrescenta ainda outra dimensão à incerteza no processo de inovação, que é a ausência de conhecimento para resolver os problemas. Com isso, a incerteza no processo inovativo não se restringe somente à ausência de informação relevante sobre a ocorrência de eventos conhecidos, mas também em limitações das capacidades computacional e cognitiva do agente para solucionar os problemas. Mesmo que a informação esteja disponível, o indivíduo apresenta limitações para reconhecer e interpretar a informação relevante.

Freeman e Soete (2008) descrevem os diversos tipos de incerteza envolvidos no processo inovativo: a incerteza técnica, a incerteza de mercado e a incerteza da economia. As incertezas técnica e de mercado são específicas ao projeto de inovação e não podem ser descontadas, eliminadas ou consideradas com um tipo de risco calculável. As incertezas técnicas referem-se ao grau em que a inovação satisfaz vários critérios técnicos, sem aumento dos custos de desenvolvimento, de produção ou de funcionamento. Portanto, as incertezas técnicas podem ser muito reduzidas nos estágios de desenvolvimento experimental e dos testes de produção.

A incerteza de mercado está relacionada à possibilidade de que a inovação não alcance sucesso no mercado. Ela se inicia na fase de comercialização, e pode ter distintas causas estando relacionado à demanda (comportamento dos consumidores), à competição (imitação) e ao tempo de entrada e de difusão (Jiang, \& Wang, 2007). A incerteza da economia, por sua vez, está presente em todos os investimentos que envolvem resultados futuros. No processo de inovação esta incerteza adquire especial importância tendo em vista que os investimentos requerem um longo período de maturação.

Freeman e Soete (2008) ainda classificam a incerteza em distintos graus, de acordo com os tipos de inovação ao qual estão associados, conforme descrito no Quadro 1. Mesmo para as inovações com níveis de incerteza mais baixos, os autores destacam que apenas uma pequena proporção da atividade de $\mathrm{P} \& \mathrm{D}$ é financiada diretamente pelo mercado de capitais. $\mathrm{O}$ financiamento do processo inovativo é realizado em grande parte através da reinversão de lucros da própria empresa, através do autofinanciamento.

\footnotetext{
${ }^{3}$ A empresa nem sempre consegue se apropriar de todo o esforço inovativo. Parte dele "transborda" para fora da empresa, gerando externalidades positivas, e mesmo quando consegue se apropriar é por diversos mecanismos que não apenas a patente. Esta é importante em alguns setores, mas em outros, estratégias mais eficazes são segredo industrial e vantagens oriundas de ser o primeiro. O trabalho seminal de Levin et. al (1987) discute estes aspectos.
} 
Quadro 1 - Diferentes graus de incerteza das atividades inovativas

\begin{tabular}{|l|l|}
\hline $\begin{array}{l}\text { Tipo de } \\
\text { incerteza }\end{array}$ & \multicolumn{1}{c|}{ Exemplo de atividade associada } \\
\hline Incerteza real & Invenção fundamental e pesquisa. \\
\hline $\begin{array}{l}\text { Incerteza muito } \\
\text { alta }\end{array}$ & $\begin{array}{l}\text { Inovação radical de produto. } \\
\text { Inovação radical de processo realizada fora da empresa. }\end{array}$ \\
\hline $\begin{array}{l}\text { Incerteza alta } \\
\text { Inovações de produto relevantes. } \\
\text { Inovação radical de processo na própria empresa. }\end{array}$ & $\begin{array}{l}\text { Inoderada } \\
\text { movas gerações de produtos existentes. }\end{array}$ \\
\hline $\begin{array}{l}\text { Incerteza baixa } \\
\text { Incerteza muito }\end{array}$ & $\begin{array}{l}\text { Inovações licenciadas; imitação de inovação de produtos. } \\
\text { Modificação em produtos e processos. } \\
\text { Adoção precoce de processos já existentes. }\end{array}$ \\
$\begin{array}{l}\text { Novo modelo; diferenciação de produto. } \\
\text { Implementação de uma nova função para produtos já existentes. } \\
\text { Adoção tardia de processos já existentes. } \\
\text { Pequenos melhoramentos técnicos. }\end{array}$ \\
\hline
\end{tabular}

Fonte: Freeman e Soete (2008).

Freeman e Soete (2008) ainda reforçam que em vista da incerteza presente no processo de inovação a maioria das empresas não possui incentivos para empreender inovações radicais, concentrando seus esforços de $\mathrm{P} \& \mathrm{D}$ em inovações defensivas ou imitativas, na diferenciação de produto e em inovação de processo, lidando, portanto, com níveis baixos de incerteza.

As empresas, até as de mesmo tamanho e que atuam no mesmo setor, possuem diferentes estruturas de custos e operam em diferentes tipos de mercados e por meio de diferentes tipos de competição. Essas diferenças afetam as suas necessidades de financiamento (Mazzucato, 2013) e justificam a existência de vários tipos de linhas de financiamento para atender aos diversos perfis de empresas. Neste contexto, o governo possui um importante papel na promoção, estímulo e apoio às empresas nos seus esforços de inovação, de forma a evitar o sub-investimento nas atividades inovativas.

Além de peculiaridades intrínsecas a sua natureza, o investimento em inovação apresenta especificidades setoriais. Diferenças setoriais na atividade inovadora têm implicações importantes para a alocação de recursos. Isso porque os diferentes tempos de duração dos projetos e volumes de investimento necessários impactam diretamente as necessidades financeiras (O’Sullivan, 2006).

O desenvolvimento de medicamentos leva em média 13 anos desde o início das atividades de pesquisa até sua comercialização (Radaelli, 2006). O setor farmacêutico é, segundo Pavitt (1984), um setor baseado em ciência, para o qual o conhecimento científico interno das empresas, das instituições de pesquisa e das universidades é relevante para a geração de inovação. No entanto, além da pesquisa para identificação de novas moléculas, novas formulações, novas apresentações, novas doses, etc, o desenvolvimento de medicamentos passa ainda por testes pré-clínicos (in vitro e/ou in vivo), testes clínicos (fase 1: para testar segurança e dosagem, fase 2: para testar eficácia e efeitos colaterais, fase 3: para monitorar reações adversas) e o registro sanitário para autorização de comercialização. Há, ainda, a fase 4 de fármaco vigilância, pós registro, para identificar efeitos colaterais e reações adversar no uso expandido do medicamento no mercado (Bastos, 2005; Radaelli, 2006). Percebe-se assim, que o volume de recursos para o desenvolvimento de um novo medicamento demanda muito mais tempo e investimento em equipamentos, insumos e 
pessoal do que o desenvolvimento de um software, por exemplo. Para o desenvolvimento de medicamentos, além dos desafios (e a incerteza) do conhecimento técnico, estão presentes ainda aspectos regulatórios durante todas as etapas do processo de desenvolvimento, registro, produção e comercialização de medicamentos. Muitas vezes, são necessários períodos extensos para conseguir as autorizações de funcionamento do estabelecimento para pesquisa e/ou produção e o registro do produto e, durante esse período, as empresas não são capazes de se sustentar com receitas próprias, e apoios financeiros para introdução da inovação no mercado são necessários.

No caso brasileiro, entre 2012 e 2014, as empresas do setor farmacêutico que implementaram inovações e que receberam apoio do governo declararam utilizar majoritariamente incentivo fiscal à $\mathrm{P} \& \mathrm{D}(42,1 \%)$ e financiamento a projetos de $\mathrm{P} \& \mathrm{D}$ e inovação tecnológica em parceria com universidades (40,5\%). A subvenção econômica $(18,6 \%)$ foi o terceiro programa mais utilizado por estas empresas. Estas demandas estão associadas aos esforços inovativos realizados pelas 191 empresas do setor $(47,1 \%)$, que no mesmo período, investiram $\mathrm{R} \$ 2,281$ bilhões, ou 4,2\% das suas receitas líquidas de vendas, em atividades inovativas, com destaque para atividades internas de P\&D (59\% das empresas inovadoras e $53,8 \%$ do valor investido), aquisição de máquinas e equipamentos $(71,1 \%$ das empresas e $15,4 \%$ do valor) e aquisição externa de P\&D (51,6\% das empresas e 9,4\% do valor) (IBGE, 2016). Os resultados da Pesquisa de Inovação a (PINTEC/IBGE)de 2014mostram que estes financiamentos foram significativos na alteração das estratégias das empresas em termos de maior propensão às atividades inovativas, em especial para o setor farmacêutico, o qual foi alvo específico das políticas e instrumentos durante toda a primeira década dos anos 2000.

Neste período, as empresas nacionais especializaram-se no desenvolvimento de medicamentos similares e genéricos (a partir da Lei 9.279/1999) ganhando uma importante participação no cenário nacional. Esta especialização, porém, reforçou o padrão nacional de esforços de inovação caracterizados por atualização e modernização, envolvendo, portanto, incerteza muito baixa, baixa e moderada, já que poucas empresas desenvolvem e implementam no país inovações disruptivas ou de fronteira. Isto porque para o desenvolvimento de medicamentos genéricos, são necessários esforços tecnológicos de adaptação de um produto que já perdeu a proteção patentária, as empresas nacionais conseguiram competir com maior facilidade neste mercado, do que em mercados que envolvem o desenvolvimento de princípios ativos inovadores a nível mundial ${ }^{4}$. No entanto, apesar de resultados ainda incipientes, o setor farmacêutico destaca-se nos dados gerais da indústria. Entre 2011-2014, 111 (52,3\%) empresas implementaram produtos novos para a empresa, $36(16,8 \%)$ empresas implementaram produtos novos para o mercado nacional e 12 $(5,7 \%)$ empresas implementaram produtos novos para o mercado mundial (IBGE, 2016). Note que as empresas nacionais são as responsáveis por grande parte destes esforços e resultados, pois as multinacionais focam a maior parte dos investimentos em suas matrizes no exterior, realizando no Brasil pesquisa para apoio ao registro e testes clínicos (Paranhos, 2012).

\footnotetext{
${ }^{4}$ Devido à importância do mercado de genéricos no Brasil, as empresas multinacionais se movimentaram para aumentar sua presença nesse mercado, por meio de fusões e aquisições. Por outro lado, as empresas nacionais ainda possuem porte menor, se comparadas com os conglomerados multinacionais, e uma penetração limitada em nichos de produtos com maior valor agregado, como o de medicamentos biológicos.
} 
Estes dados evidenciam, portanto, esforços do setor farmacêutico brasileiro de incremento do grau de incerteza das atividades de inovação refletidas no desenvolvimento de produtos novos para o mercado nacional e mundial. Este trabalho se propõe a analisar em qual medida este aumento pode ser atribuído ao instrumento da subvenção econômica da FINEP, que por suas características de recurso não-reembolsável deveria ser utilizado no desenvolvimento de projetos de inovação mais arriscados e incertos.

\section{A Subvenção Econômica na FINEP}

A Finep foi criada em 1967 com a finalidade de financiar estudos e programas necessários à definição dos projetos de modernização e industrialização correntes na época. Em 1969 foi criado o Fundo Nacional de Desenvolvimento Científico e Tecnológico FNDCT com a finalidade de dar apoio financeiro aos programas e projetos prioritários de desenvolvimento científico e tecnológico. A FINEP atua como secretária executiva do FNDCT. O FNDCT, através da FINEP, juntamente com o BNDES constitui as principais fontes de financiamento à ciência, tecnologia e inovação no Brasil.

No Brasil, os subsídios para as empresas na modalidade de subvenção econômica são relativamente recentes tendo sido instituídos, ainda de forma restrita, pela Lei 10.332/2001 às empresas com Programa de Desenvolvimento Tecnológico Industrial (PDTI) ou Programa de Desenvolvimento Tecnológico Agropecuário (PDTA). Entretanto, somente com a implementação da Lei 10.973/2004 (Lei de Inovação), e seu Decreto Regulamentar 5.563/2005, que a subvenção econômica (financiamento não-reembolsável à empresa) é implementado para atividades de pesquisa, desenvolvimento tecnológico e inovação com objetivo de desenvolvimento de produtos e processos inovadores nas empresas nacionais, com especial atenção ao atendimento às micro e pequenas empresas. A subvenção econômica é então instituída com recursos orçamentários no âmbito do Fundo Nacional para o Desenvolvimento Científico e Tecnológico (FNDCT) (Koeller, 2009). Sendo assim, a operacionalização destes recursos não-reembolsáveis se iniciou de forma efetiva em 2006 pela FINEP por meio de chamadas públicas do Programa de Subvenção Econômica.

A Lei $n^{0} 11.196 / 05$, por sua vez, institui outra modalidade de subvenção que é a concessão de recursos para que as empresas, localizadas no território brasileiro, contratassem pesquisadores a serem envolvidos em atividades de inovação. A subvenção poderia ser de até $60 \%$ do valor da remuneração de pesquisadores mestres ou doutores.

Uma primeira iniciativa da FINEP na subvenção foi o Programa de Apoio à Pesquisa em Empresas (PAPPE-Integração) instituído em 2003 em parceria com as fundações de amparo a pesquisas estaduais. O Programa PAPPE era destinado a financiar atividades de $\mathrm{P} \& \mathrm{D}$ de produtos e processos inovadores realizados por pesquisadores atuantes diretamente ou em cooperação com empresas de base tecnológica. O programa apoiou projetos nas etapas anteriores à de comercialização, financiando em uma primeira etapa estudos de viabilidade técnica e econômica e em uma segunda etapa o desenvolvimento final de novos produtos e processos para a colocação no mercado. Os aportes máximos da FINEP eram de R $\$ 50.000,00$ na primeira fase e de até $\mathrm{R} \$ 150.000,00$ na segunda fase.

A partir de 2006, a FINEP lançou três editais com modalidades diferentes de apoio para a subvenção econômica, sendo os mesmos: (a) subvenção Econômica para o desenvolvimento de P\&D nas empresas; (b) subvenção para incorporar pesquisadores nas empresas (Programa Subvenção-Pesquisador na Empresa); (c) subvenção econômica para micro e pequenas empresas através de instituições locais (Programa Pappe-Subvenção). Em 
2008 foi lançado o Programa Prime, uma modalidade de subvenção destinada às empresas nascentes.

O primeiro edital de Subvenção Econômica foi lançado em 2006 pela FINEP, sendo lançados editais anualmente até 2010. Os editais seguintes foram lançados em 2013 e foram temáticos, havendo editais separados para as diferentes áreas. De modo geral, os editais contêm as áreas priorizadas, os requisitos dos participantes, informações sobre os recursos disponíveis, as características da proposta e os critérios para sua seleção, bem comoo cronograma da chamada, além de disposições gerais. O processo de avaliação e os critérios de seleção variam de acordo com cada edital. De modo geral, primeiramente se faz uma análise documental, para checar se todas as exigências formais foram cumpridas, e em seguida a proposta é analisada por comitês de especialistas, tanto da FINEP quanto consultores externos. Em relação à avaliação da proposta em si, geralmente os requisitos da proposta abrangem critérios pertinentes ao mérito da inovação, os aspectos mercadológicos e à capacidade de execução e os aportes da empresa. A partir de 2013, os recursos de subvenção econômica passaram a ser alocados dentro do Programa Plano Inova Empresa que abrange os mecanismos de apoio do BNDES, da FINEP e de outros órgãos públicos participantes, em contratos de apoio mistos denominados Planos Conjuntos. Foram lançados diversos editais temáticos no contexto do Inova Empresa, sendo um deles o Inova Saúde.

A Tabela 1 apresenta o número de projetos e os valores aprovados no Programa Subvenção Econômica da FINEP entre 2007 e 2014. Observa-se uma variação significativa no número de projetos apoiados ao longo dos anos, uma diminuição significativa do número de projetos selecionados, e, por sua vez, um aumento no valor médio dos projetos apoiados. $\mathrm{O}$ valor dos projetos aprovados por ano diminui significativamente ao longo dos anos, com uma retomada no aumento dos valores a partir de 2013.

Tabela 1 - Número de projetos e valores aprovados no Programa Subvenção Econômica da FINEP, 2007 a 2014.

\begin{tabular}{|l|c|c|c|}
\hline Ano & $\begin{array}{c}\text { Númerode } \\
\text { projetos }\end{array}$ & $\begin{array}{c}\text { Valor aprovado (R\$ } \\
\text { milhões) }\end{array}$ & $\begin{array}{c}\text { Valor médio por projeto } \\
\text { (R\$ milhões) }\end{array}$ \\
\hline 2007 & 218 & 520,9 & 2,39 \\
\hline 2008 & 139 & 394,5 & 3,75 \\
\hline 2009 & 213 & 433,6 & 2,45 \\
\hline 2010 & 252 & 517,0 & 2,07 \\
\hline 2011 & 90 & 192,5 & 5,79 \\
\hline 2012 & 48 & 64,1 & 10,85 \\
\hline 2013 & 37 & 120,0 & 14,08 \\
\hline 2014 & 78 & 252,8 & 6,68 \\
\hline
\end{tabular}

Fonte: FINEP, elaboração própria (2020).

\section{Metodologia}

Este trabalho valeu-se de uma pesquisa qualitativa e descritiva abrangendo diversos casos sendo analisados diferentes projetos de subvenção econômico da FINEP. Os estudos multicaso permitem levantar evidências para um número maior de casos, o que repercute em maior confiabilidade do que se fosse apenas um caso (Yin, 2001). Este tipo de abordagem caracteriza-se pelo estudo de variados objetos de forma profunda para se inferir hipóteses. No caso deste trabalho a hipótese a ser observada é se os projetos de subvenção econômica 
apoiados pela FINEP do setor farmacêutico são de elevados níveis de incerteza. Uma característica desta metodologia é que os resultados encontrados para alguns casos não podem ser generalizados para a população. Um dos objetivos deste tipo de trabalho é identificar informações que permitam uma ação ou predição (Roesch, 1999).

A relação dos projetos apoiados pela FINEP no período de 2007 a 2014 foi obtida diretamente com a agência através da Lei de Acesso à Informação (LAI) e traz diversas informações sobre os projetos como proponente, CNAE, localidade, data de assinatura e término, valor contratado e título. Estes dados foram complementados com os dados disponibilizados pela FINEP na seção Transparência - Projetos Contratados em seu sítio eletrônico ${ }^{5}$, pelo Sistema de Informações Gerenciais de Ciência Tecnologia e Inovação SIGCTI $^{6}$ e outras informações não disponíveis nestas bases de dados, solicitadas também via $\mathrm{LAI}^{7}$, como o objetivo e resumo dos projetos contratados. Este processo de pesquisa aconteceu entre o final de 2016 e início de 2017.

A classificação dos projetos do setor farmacêutico quanto a seu nível de incerteza foi realizada de acordo com o Quadro 2. Foram listados e classificados os tipos gerais de projetos de inovação realizados no setor, e estes receberam denominações genéricas. A importância dessa generalização consiste em padronizar a avaliação, de forma a obter um instrumento capaz de classificar tipos diversos de projetos e homogeneizar a descrição. Além disso, esta classificação é uma tentativa de avaliar os projetos principalmente de acordo com sua incerteza tecnológica, não sendo possível avaliar significativamente, a partir das informações disponíveis, outros aspectos como: potencial de mercado, importância econômica e social, dentro outros.

De modo a reduzir a subjetividade da classificação, após a listagem e classificação inicial, o quadro foi revisto e validado por diversos especialistas do setor ${ }^{8}$ provenientes de diferentes áreas e institutos de pesquisa, tanto relacionadas a saúde e políticas públicas, quanto a áreas diretamente ligadas ao desenvolvimento de produtos farmacêuticos, como toxicologia e fito medicamentos. Cada especialista realizou sua revisão de forma independente e de acordo com sua própria experiência, mas em um mesmo intervalo de tempo, e coube aos autores compilar as sugestões para a elaboração e formatação do Quadro 2. Os especialistas fizeram sugestões relativas à inclusão de atividades e sua descrição e ao nível de incerteza aplicável a elas. Por fim, este quadro foi novamente enviado aos especialistas, para que pudessem fazer a validação final.

A cada nível de incerteza foi atribuído um número inteiro, variando de 1 a 5 (vide última coluna do Quadro 2), sendo 1 correspondente à categoria de menor incerteza e 5 à categoria de maior incerteza. Para a classificação do projeto foi imprescindível algumas informações mínimas sobre seu escopo, para se evitar uma classificação incoerente. Sendo

\footnotetext{
${ }^{5}$ http://finep.gov.br/acesso-a-informacao-externo/transparencia/projetos-contratados

${ }^{6}$ http://sigcti.mct.gov.br/

${ }^{7}$ O pedido de informações via LAI aconteceu em 2016 e a complementação de informações dos resumos dos projetos foi solicitada em janeiro de 2017.

${ }^{8}$ Os revisores foram Carlos Alberto Tagliati, pesquisador de Toxicologia da Faculdade de Farmácia da UFMG; Gabriela Chaves Costa, pesquisadora do Núcleo de Assistência Farmacêutica da Escola Nacional de Saúde Pública da Fundação Oswaldo Cruz; Cristiane Mota Soares, tecnologista em Saúde Pública na área de Fito medicamentos em Farmanguinhos na Fundação Oswaldo Cruz; Josiely Gomes, Mestre em Bioquímica e Imunologia pela UFMG; Renan Gadoni Canaan, mestre em Inovação Tecnológica e Propriedade Intelectual pela UFMG. Os autores foram responsáveis pela compilação das sugestões e elaboração final do quadro
} 
assim, foi possível classificar apenas os projetos cujo objetivo ou resumo são foram conhecidos. Não foi possível obter tais informações para 34 projetos, pois, apesar de solicitado por meio da LAI, o pedido para estes projetos foi negado sob alegação de proteção legal ao segredo comercial ou de empresa. Para apenas um projeto com o resumo a descrição não foi detalhada o suficiente para determinar o nível de incerteza.

Quadro 2 - Nível de incerteza no setor farmacêutico

\begin{tabular}{|c|c|c|}
\hline $\begin{array}{l}\text { Nível de } \\
\text { incerteza }\end{array}$ & Tecnologia & Nota \\
\hline Muito alta & $\begin{array}{l}\text { - Desenvolvimento de uma nova molécula com ação em um novo alvo molecular, } \\
\text { objeto de patente, que seja o primeiro de uma indicação terapêutica ou que traga } \\
\text { ganho para o tratamento em relação aos medicamentos já utilizados para a uma } \\
\text { indicação terapêutica, com a comprovação de maior eficácia em relação aos } \\
\text { medicamentos existentes para a mesma; mesma eficácia com diminuição significativa } \\
\text { dos efeitos adversos; ou mesma eficácia com redução significativa do custo global de } \\
\text { tratamento. } \\
\text { - Desenvolvimento de medicamentos biológicos novos e seu processo de produção. }\end{array}$ & 5 \\
\hline Alta & $\begin{array}{l}\text { - Desenvolvimento de nova molécula com ação em um alvo já estabelecido que seja } \\
\text { objeto de patente e que traga ganho para o tratamento em relação aos medicamentos } \\
\text { já utilizados para a mesma indicação terapêutica, com a comprovação de maior } \\
\text { eficácia em relação aos medicamentos existentes para a mesma indicação terapêutica; } \\
\text { mesma eficácia com diminuição significativa dos efeitos adversos; ou mesma } \\
\text { eficácia com redução significativa do custo global de tratamento. } \\
\text { - Desenvolvimento de um novo medicamento fitoterápico. } \\
\text { - Prospecção de novos alvos moleculares: genes, biomarcadores, epílogos, receptores, } \\
\text { dentre outros. } \\
\text { - Novo processo biológico ou melhoria de processo existente para a obtenção de } \\
\text { moléculas já conhecidas. }\end{array}$ & 4 \\
\hline Moderada & $\begin{array}{l}\text { - Desenvolvimento de um novo medicamento e/ou de um novo processo de produção } \\
\text { para moléculas novas para a empresa, mas já comercializadas no mercado nacional } \\
\text { ou internacional. } \\
\text { - Nova formulação de princípio ativo já conhecido utilizando sistema de delivery } \\
\text { inovador. } \\
\text { - Desenvolvimento de um novo sistema de delivery baseado em tecnologias } \\
\text { disruptivas, por exemplo, nanotecnologia. } \\
\text { - Validação farmacológica de insumos farmacêuticos ativos vegetais para } \\
\text { fitoterápicos e produtos tradicionais fitoterápicos (baseados em etnobotânica, uso } \\
\text { popular e tradicional). } \\
\text { - Caracterização e pesquisa relacionada a marcadores moleculares já identificados. } \\
\text { - Nova associação de princípios ativos já comercializados no país. } \\
\text { - Desenvolvimento de novo processo de síntese e produção de moléculas já } \\
\text { conhecidas. }\end{array}$ & 3 \\
\hline Baixa & $\begin{array}{l}\text { - Desenvolvimento de um medicamento genérico. } \\
\text { - Desenvolvimento de novo sistema de drug delivery baseado em tecnologias já } \\
\text { estabelecidas/tradicionais. } \\
\text { - Nova formulação de princípio ativo já conhecido utilizando sistemas de delivery já } \\
\text { estabelecidos. } \\
\text { - Nova formulação de medicamentos fitoterápicos já disponíveis no mercado. } \\
\text { - Caracterização dos insumos farmacêuticos ativos vegetais para fitoterápicos e } \\
\text { produtos tradicionais fitoterápicos (baseados em etnobotânica, uso popular e } \\
\text { tradicional). Inclui análises físico-químicas; desenvolvimento e validação de métodos } \\
\text { analíticos, elaboração das monografias farmacopéicas. } \\
\text { - Medicamento já comercializado pela empresa, em nova forma farmacêutica. }\end{array}$ & 2 \\
\hline
\end{tabular}




\begin{tabular}{|c|c|c|}
\hline \multicolumn{3}{|c|}{ Quadro 2 - Nível de incerteza no setor farmacêutico (continuação) } \\
\hline $\begin{array}{l}\text { Nível de } \\
\text { incerteza }\end{array}$ & Tecnologia & Nota \\
\hline $\begin{array}{l}\text { Muito } \\
\text { baixa }\end{array}$ & $\begin{array}{l}\text { - Adequação de matérias primas fundamentais em um produto que requeiram certo } \\
\text { esforço tecnológico. } \\
\text { - Desenvolvimento de medicamento fitoterápico para registro simplificado na } \\
\text { ANVISA. } \\
\text { - Melhoramento das espécies vegetais utilizadas em produtos naturais envolvendo } \\
\text { seleção das melhores condições de cultivo, melhoramento genético tradicional, } \\
\text { visando melhorar o rendimento da planta. } \\
\text { - Implantação de unidade fabril e/ou laboratório de pesquisa e desenvolvimento no } \\
\text { setor farmacêutico. } \\
\text { - Nova apresentação de medicamento já comercializado pela própria empresa, em } \\
\text { uma mesma forma farmacêutica. }\end{array}$ & 1 \\
\hline
\end{tabular}
Fonte: Elaboração dos autores a partir de Freeman e Soete (2008).

\section{Análise do aporte de Recursos de Subvenção Econômica pela FINEP no Setor Farmacêutico de 2007 a 2014}

\section{a. Panorama Geral}

O montante de recursos de subvenção investido pela FINEP no setor farmacêutico no período analisado foi de $\mathrm{R} \$ 210,6$ milhões, o que corresponde a 8,4\% do montante total de recursos aportados em subvenção pela agência. Este montante foi aportado em 87 projetos e 60 empresas foram contempladas, o que mostra que algumas empresas contrataram mais de um projeto. A Tabela 2 traz um resumo destes dados.

Tabela 2 - Número de projetos e montante investido na subvenção econômica pela FINEP, 2007-2014.

\begin{tabular}{|c|c|l|l|l|}
\hline Setor & Número de projetos & \% & \multicolumn{1}{c|}{ Total } & \% \\
\hline Farmacêutico & 87 & 8,1 & $\mathrm{R} \$ 210.636 .558,53$ & 8,4 \\
\hline Outros Setores & 988 & 91,9 & $\mathrm{R} \$ 2.284 .717 .205,71$ & 91,6 \\
\hline Total & 1075 & 100,0 & $\mathrm{R} \$ 2.495 .353 .764,24$ & 100,0 \\
\hline
\end{tabular}

Fonte: Elaboração própria a partir de dados da FINEP (2017).

O Gráfico 1 apresenta a distribuição dos recursos aportados nos projetos do setor farmacêutico em relação ao total do investimento em subvenção econômica. Como pode ser visualizado no gráfico, há uma grande variação ao longo dos anos. O setor farmacêutico foi considerado estratégico em todas as políticas industriais recentes (Teixeira, et. al. 2017), chegando a receber um quinto dos recursos de subvenção da FINEP em 2012. 
Gráfico 1 - Investimento setorial - Investimento em subvenção no setor farmacêutico em relação ao investimento em subvenção total (\% 2007-2014)

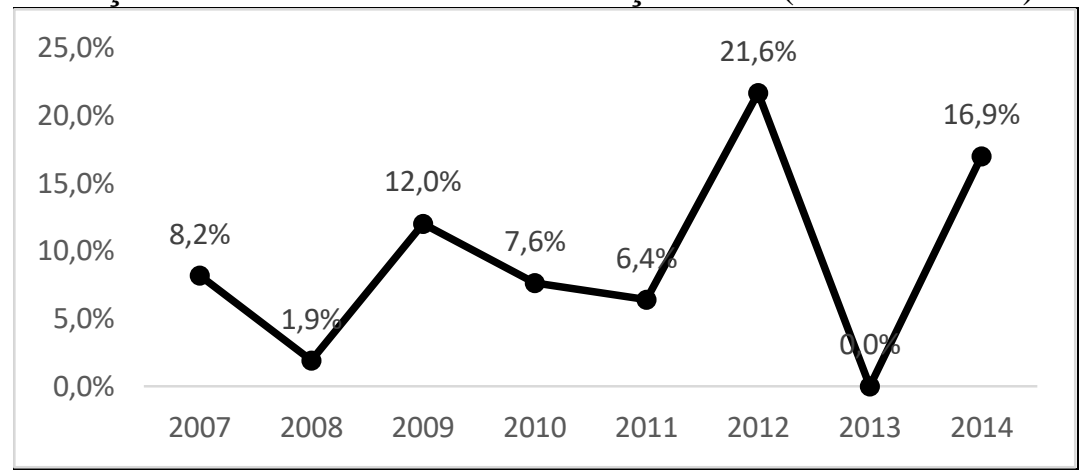

Fonte: Elaboração própria a partir de dados da FINEP (2020).

Os projetos de subvenção do setor farmacêutico foram selecionados por meio de 8 (oito) editais lançados em diferentes anos. São eles os editais Subvenção Econômica à Inovação de 2006, 2007, 2008, 2009 e 2010 que contemplava várias áreas do conhecimento e o edital subvenção de 2013 que passou a ser temático, sendo para este trabalho o dedicado a área de biotecnologia. Entre os anos de 2006 e 2007 houve também o edital Pesquisador na Empresa, já em 2013 houve o edital Inova Saúde, lançado no contexto do programa Inova Empresa. A Tabela 3 sumariza estas informações, apresentando também o número de projetos contratados por edital.

Tabela 3- Ano e número de projetos do setor farmacêutico contratados por edital, 2006 - 2013

\begin{tabular}{|c|c|c|}
\hline Ano & Edital & $\begin{array}{l}\text { Número de projetos } \\
\text { contratados }\end{array}$ \\
\hline $2006 / 2007$ & Pesquisador na empresa & 5 \\
\hline 2006 & $\begin{array}{l}\text { Subvenção econômica à inovação } 2006 \text { (Áreas: Biotecnologia, } \\
\text { Fármacos e Medicamentos e Geral) }\end{array}$ & 8 \\
\hline 2007 & Subvenção econômica à inovação 2007 & 8 \\
\hline 2008 & Subvenção econômica à inovação 2008 & 28 \\
\hline 2009 & $\begin{array}{l}\text { Subvenção econômica à inovação } 2009 \text { (Áreas: Saúde e } \\
\text { Biotecnologia) }\end{array}$ & 20 \\
\hline 2010 & $\begin{array}{l}\text { Subvenção econômica à inovação } 2010 \text { (Áreas: Saúde e } \\
\text { Biotecnologia) }\end{array}$ & 10 \\
\hline 2013 & Subvenção econômica à inovação 2013 - Biotecnologia & 1 \\
\hline 2013 & Inova Saúde & 7 \\
\hline \multicolumn{2}{|r|}{ Total } & 87 \\
\hline
\end{tabular}

Fonte: Elaboração própria a partir de dados da FINEP (2017).

A maioria dos recursos foi destinada a região Sudeste, principalmente ao estado de São Paulo, que recebeu 63,1\% dos recursos (Gráfico 2) no período analisado. Essa distribuição reflete a concentração das indústrias farmacêuticas neste estado e no Rio de Janeiro, segunda maior aglomeração de indústrias farmacêuticas do país (Paranhos, et al., 2015). A região Centro Oeste, que cresceu significativamente nos últimos anos em número de empresas do setor instaladas, foi a terceira região que mais recebeu recursos $(5,8 \%)$, sendo precedida pela região Sul que recebeu $14,7 \%$ dos recursos. 
Gráfico 2 - Distribuição dos recursos de subvenção econômica para projetos do setor farmacêutico por UF, 2007-2014

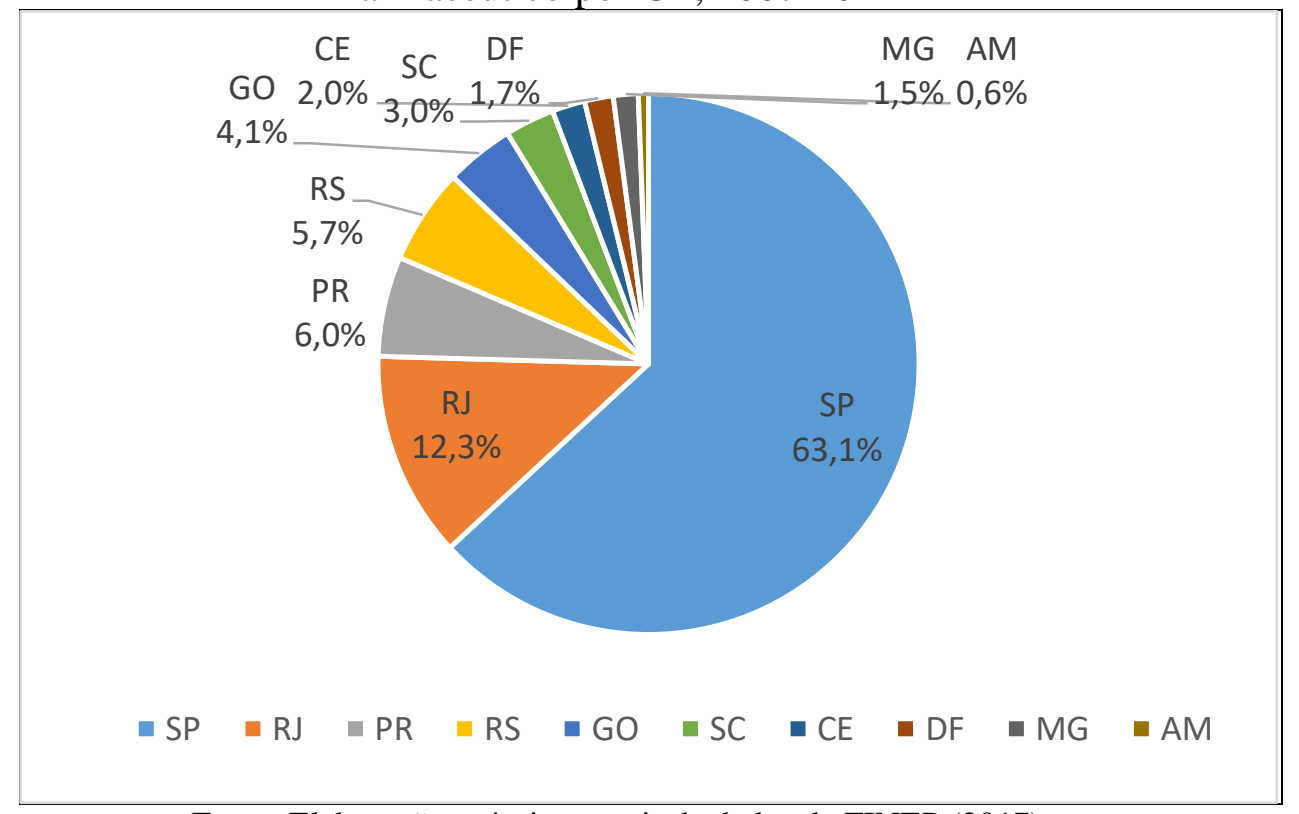

Fonte: Elaboração própria a partir de dados da FINEP (2017).

Apesar da maioria das empresas $(81,7 \%)$ ter contratado apenas um projeto algumas empresas receberam recursos mais de uma vez em diferentes projetos de subvenção. A Tabela 4 mostra o número de empresas por número de projetos contratados. As empresas com maior número de projetos contratados foram a Cristália, localizada em SP, e a Nortec Química, localizada no RJ, com 9 e 5 projetos respectivamente. Cabe citar que o Laboratório Cristália executa tanto a fabricação de medicamentos quanto a fabricação de farmoquímicos ${ }^{9}$, enquanto a Nortec é uma das principais produtoras de farmoquímicos do Brasil. Ambas as empresas possuem projetos de desenvolvimento de antirretrovirais, contratados em 2007, e a Cristália tem diversos projetos na área de medicamentos biológicos a partir de 2011 (Prochnik, et. al. 2016), resultado dos projetos de transferência de tecnologia da política de Parcerias de Desenvolvimento Produtivo (PDP) do Ministério da Saúde (Ministério da Saúde, 2018).

Tabela 4 - Número de projetos do setor farmacêutico contratados por empresa, 20072014

\begin{tabular}{|c|c|}
\hline Número de projetos contratados & Número de empresas \\
\hline 9 projetos contratados & 1 \\
\hline 5 projetos contratados & 1 \\
\hline 3 projetos contratados & 6 \\
\hline 2 projetos contratados & 3 \\
\hline 1 projeto contratado & 49 \\
\hline Total & 60 \\
\hline
\end{tabular}

Fonte: Elaboração própria a partir de dados da FINEP (2017). 
b. Análise Temporal

O valor investido pela FINEP no setor farmacêutico apresentou variação nos anos analisados, como mostra o Gráfico 3. O ápice de projetos e recursos aconteceu em 2009, com $\mathrm{R} \$ 52,1$ milhões de recursos investidos em 29 projetos. Tais projetos foram contratados neste ano, porém foram selecionados em um edital anterior a essa data - o Edital de Subvenção Econômica de 2008 - o que indica que os investimentos públicos em inovação para este setor não foram afetados pela crise mundial que ocorreu entre 2008 e 2009. Os efeitos se pronunciam de maneira mais acentuada nos anos 2011 e 2012, onde houve uma diminuição relevante nos recursos públicos não-reembolsáveis investidos. Isso pode ser explicado pela ausência de editais de subvenção no ano de 2011, sendo que os projetos assinados nesses dois anos correspondem a projetos selecionados no edital de subvenção de 2010. Não houve nenhum projeto contratado em 2013, mas como foram lançados dois editais neste ano, alguns projetos foram contratados em 2014.

Gráfico 3 - Distribuição dos recursos por ano e número de projetos do setor farmacêutico apoiados entre 2007-2014 (R milhões)

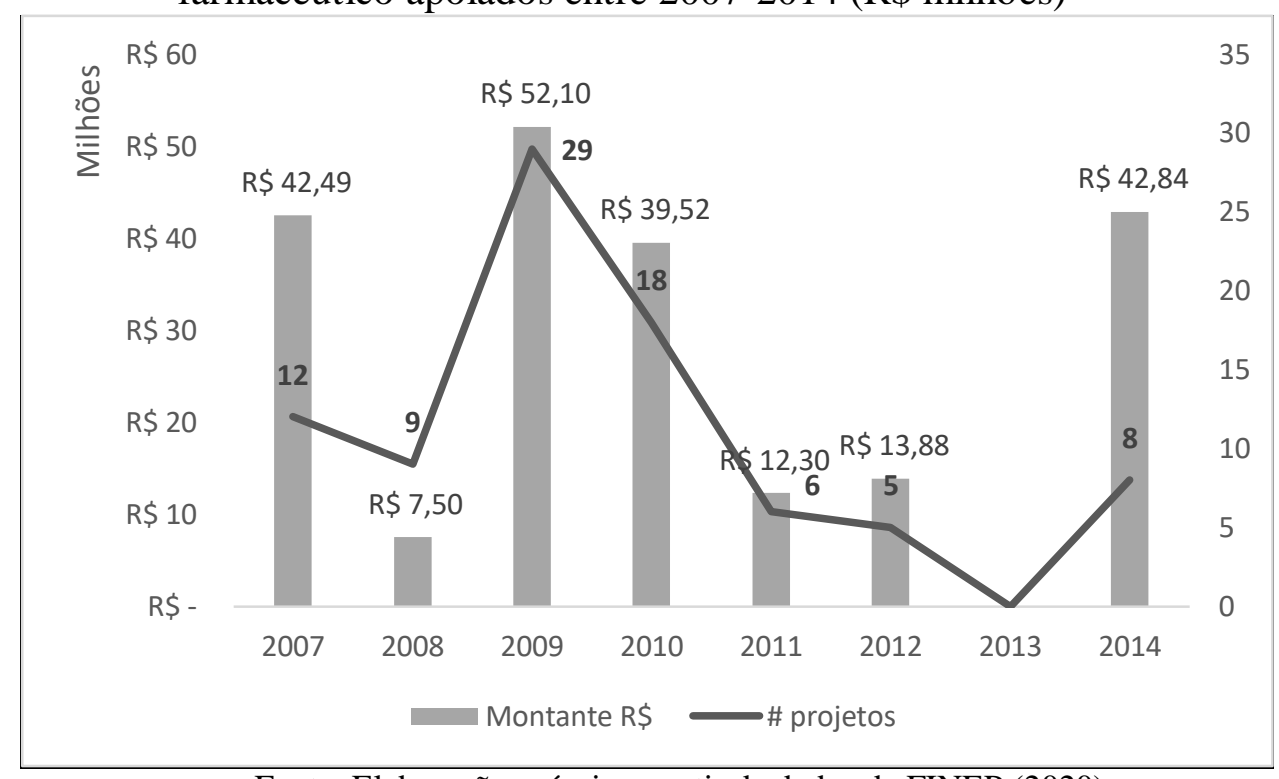

Fonte: Elaboração própria a partir de dados da FINEP (2020).

O valor médio dos projetos apresenta uma tendência crescente, atingindo um ápice em 2014, como mostra o Gráfico 4. O alto valor médio dos projetos, de R \$ 2,4 milhões considerando todos os anos, reflete o alto volume de recursos necessários para $o$ desenvolvimento de tecnologias neste setor. 
Gráfico 4 - Distribuição do valor médio dos projetos de subvenção do setor farmacêutico, 2007-2014 (R \$ milhões)

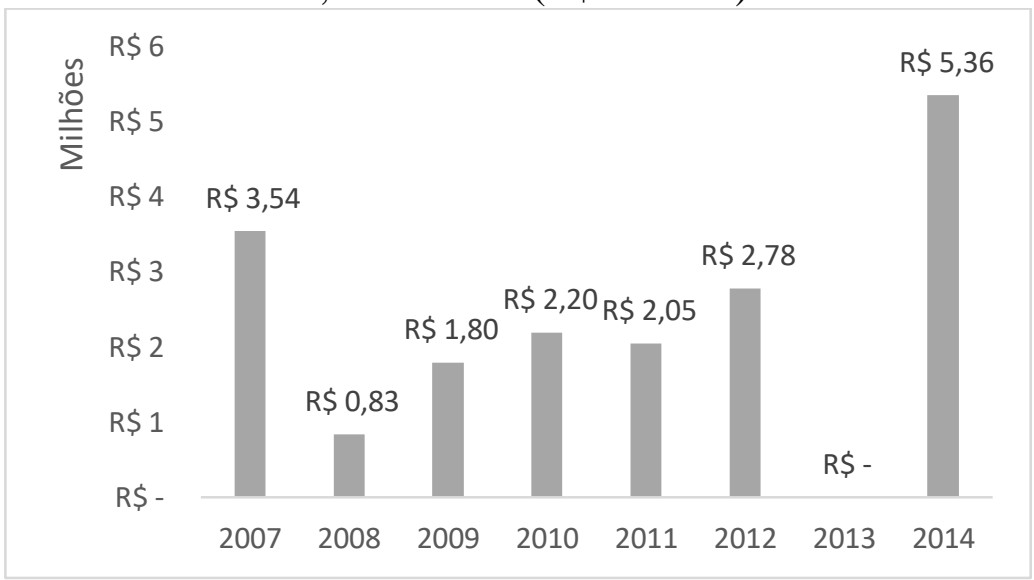

Fonte: Elaboração própria a partir de dados da FINEP.

\section{c. Nível de Incerteza Tecnológica dos Projetos do Setor Farmacêutico Subvencionados}

Esta seção apresenta os projetos de subvenção de acordo com o grau de incerteza, conforme classificação descrita na seção 3. "Metodologia". A Tabela 5 apresenta a distribuição das notas e a quantidade de projetos da área farmacêutica classificados em cada categoria.

Dentre os 52 projetos classificados, 20 (ou $23 \%$ do total)foram classificados como de alta incerteza, 21 (ou 24,1\%) como de incerteza moderada, $9(10,3 \%)$ com baixa incerteza e 2 $(2,3 \%)$ de baixa incerteza. Portanto, os projetos receberam nota média de $3,13^{10}$, correspondente a uma incerteza moderada. Os projetos de incerteza alta corresponderam a novo processo biológico ou melhoria de processo existente para a obtenção de moléculas já conhecidas ou desenvolvimento de nova molécula com ação em um alvo já estabelecido que seja objeto de patente e que traga ganho para o tratamento em relação aos medicamentos já utilizados para a mesma indicação terapêutica, com a comprovação de maior eficácia em relação aos medicamentos existentes para a mesma indicação terapêutica; mesma eficácia com diminuição significativa dos efeitos adversos; ou mesma eficácia com redução significativa do custo global de tratamento.

\footnotetext{
${ }^{10}$ A incerteza média foi calculada a partir da média ponderada. Os valores seriam o número designado para cada nível de incerteza (de 1 a 5) e os pesos corresponde ao número de projetos em cada nível de incerteza.
} 
Tabela 5 - Nível de incerteza e a nota atribuída a eles, e o número de projetos classificados em cada categoria, de 2007 a 2014

\begin{tabular}{|c|c|c|c|}
\hline Nível de incerteza & Nota & \# projetos & $\begin{array}{c}\text { \% sobre o } \\
\text { total de } \\
\text { projetos }\end{array}$ \\
\hline Muito alta & 5 & 0 & $0 \%$ \\
\hline Alta & 4 & 20 & $23 \%$ \\
\hline Moderada & 3 & 21 & $24,1 \%$ \\
\hline Baixa & 2 & 9 & $10,3 \%$ \\
\hline Muito baixa & 1 & 2 & $2,3 \%$ \\
\hline Notal parcial & - & $\mathbf{5 2}$ & $\mathbf{5 9 , 8 \%}$ \\
\hline Indeterminado foi possível determinar pelo resumo/objetivo & - & 1 & $1,2 \%$ \\
\hline Total Geral & - & 34 & $38,6 \%$ \\
\hline
\end{tabular}

Fonte: Elaboração própria a partir de dados da FINEP (2017).

É interessante observar a variação da nota média de incerteza por ano, como evidenciado no Gráfico 5. Observa-se uma redução do nível médio de incerteza dos projetos de subvenção dos anos de 2008 e 2009, o que é coincidente com o período de crise mundial. Depois deste período, apesar de flutuações, a nota média de incerteza se mantém superior.

Gráfico 5 - Nota média de incerteza por ano, de 2007 a 2014

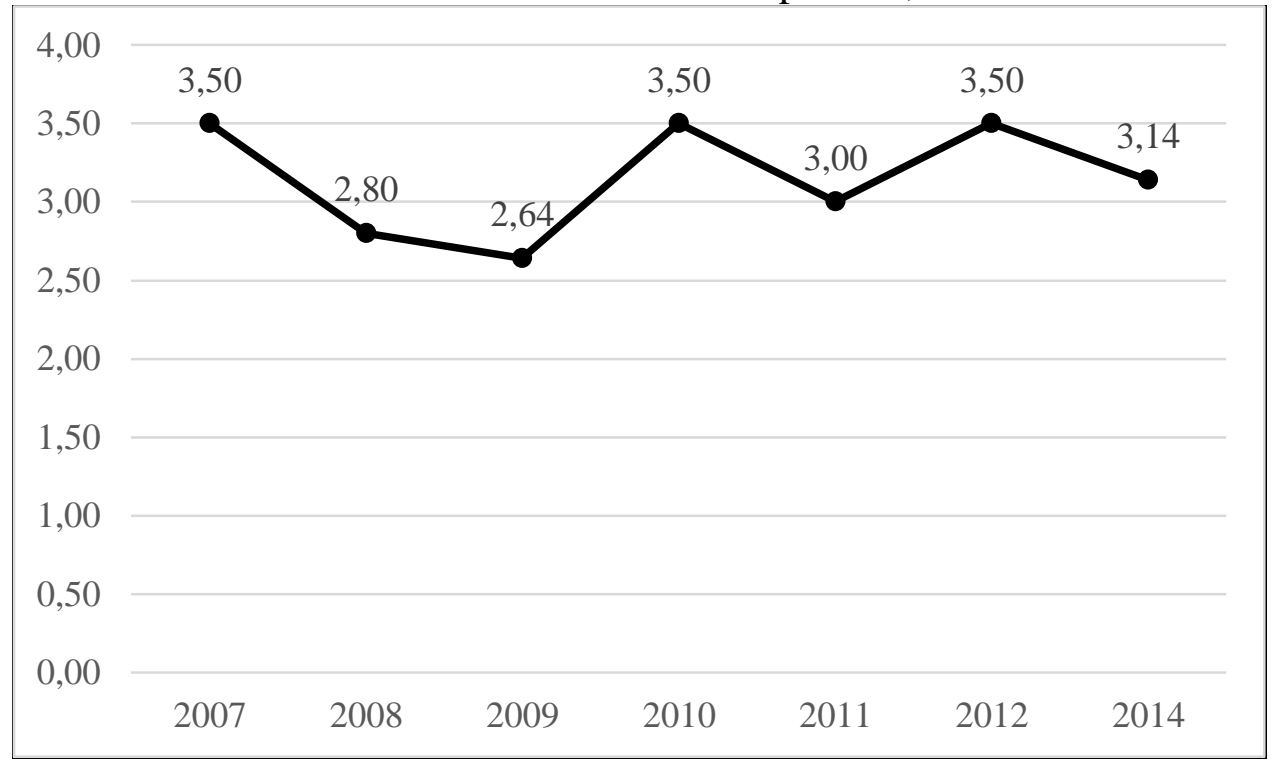

Fonte: Elaboração própria a partir de dados da FINEP (2017).

\section{Conclusão}

O presente trabalho apresentou um esforço em avaliar os projetos farmacêuticos de subvenção apoiados pela FINEP entre 2007-2014 de acordo com o grau de incerteza, seguindo metodologia desenvolvida em Cannan (2016). Para isto foram analisados dados obtidos por meio da LAI e envolveu um esforço multidisciplinar de buscar junto à especialistas do setor a construção da metodologia para a classificação dos projetos de subvenção econômica.

A subvenção econômica para as empresas é um instrumento relativamente recente no Brasil, carecendo de estudos de avaliem seus resultados no fomento ao processo de inovação. 
Este trabalho se propôs a identificar e analisar uma dimensão ainda pouco explorada que é a que concerne à análise ex-post do nível de incerteza envolvido nos projetos subvencionados. Como a subvenção se caracteriza por disponibilizar recursos não reembolsáveis, a principal recomendação é que este instrumento seja utilizado nos projetos ou etapas de maior incerteza e risco. Os resultados indicaram que $23 \%$ dos projetos apoiados foram de alta incerteza, não havendo projetos de incerteza muito alta. Neste sentido, o trabalho corrobora resultado de Costa et al. (2013), que analisou 145 projetos de subvenção de diferentes áreas de 2006, 2007 e 2008 e encontrou a falta de foco em projetos mais inovadores.

Conforme apresentado, as empresas farmacêuticas vêm ampliando seus investimentos em atividades internas de $\mathrm{P} \& \mathrm{D}$, bem como seus esforços na criação de conhecimentos novos e na acumulação tecnológica entre 2006 e 2014 em decorrência de um conjunto de instrumentos públicos de apoio à inovação como o Profarma do BNDES e as Parcerias de Desenvolvimento Produtivo (Paranhos, et al., 2020). Estes esforços, em alguma medida, serão acompanhados de uma maior capacidade de propor projetos de maior complexidade e maior nível de incerteza, que podem ser potencializados por editais de financiamento a projetos de maior risco e incerteza, como é o caso da subvenção econômica.

Visto o prazo de maturação de estratégias inovativas neste setor, o período de análise deste trabalho pode não ter captado a mudança nas estratégias de inovação em termos de complexidade e incerteza por parte das empresas farmacêuticas brasileiras. A subvenção econômica é uma modalidade de financiamento relevante para incentivar projetos maior incerteza, podendo ter efeitos bastante positivos para o desenvolvimento produtivo e inovativo da indústria farmacêutica brasileira. Desta forma, se está potencializando a aplicação dos recursos públicos no fomento à inovação, aplicando em tecnologias de maior risco, que não seriam desenvolvidas individualmente pelas empresas, caso o apoio não existisse (Rosenberg, 1996).

No entanto, a drástica redução dos recursos destinados a estas atividades e o desmantelamento dos instrumentos de estímulo ao desenvolvimento da indústria farmacêutica desde 2016, colocam em risco os investimentos públicos e privados realizados e a capacidade de resposta da indústria local no enfrentamento da forte dependência externa presente no Brasil nesta indústria em particular, e na área de saúde, em geral (Paranhos, et al., 2020).

Uma importante limitação do trabalho quanto à classificação dos projetos em relação ao nível de incerteza é que ela foi realizada a partir do resumo dos projetos. O resumo contém informações reduzidas e simplificadas. Ademais foi possível classificar somente $61 \%$ dos projetos subvencionados no período analisado, visto que o acesso ao resumo dos demais projetos foi negado alegando-se proteção às informações das empresas. Os demais $39 \%$ dos projetos podem apresentar um padrão distinto do encontrado neste trabalho, em termos do nível de incerteza, limitando as inferências realizadas. Os resultados encontrados, portanto, não podem ser generalizados para os projetos de subvenção apoiados pela FINEP no período deste estudo, ficando o estímulo para que a própria agência faça esta analise a partir da metodologia desenvolvida neste trabalho.

\section{Referências}

Arrow, K. J. (1962). Economic Welfare and the Allocation of Resources for Invention. In: Nelson, R. (1962). The Rate and Direction of Inventive Activity. NBER: Princeton University Press, 609-629. 
Bastos, V. (2005). Inovação Farmacêutica: Padrão Setorial e Perspectivas para o Caso Brasileiro. BNDES Setorial, Rio de Janeiro, 22, 271-296.

Brasil. (2014). Instituto Brasileiro de Geografia e Estatística - IBGE. Pesquisa de Inovação. Rio de Janeiro: IBGE. Disponível em: <http://www.pintec.ibge.gov.br/>. Acesso em: 23 de junho de 2017.

Brasil. (2018). Ministério da Saúde. Parceria para o Desenvolvimento Produtivo. Ministério da Saúde, Brasília. Disponível em http://portalms.saude.gov.br/ciencia-etecnologia-e-complexo-industrial/complexo-industrial/parceria-para-o-desenvolvimentoprodutivo-pdp.

Canaan, R.G. (2016). Financiamento à Inovação em Biotecnologia: Uma Avaliação da Atuação da FINEP no Aporte de Recursos Não-Reembolsáveis. Dissertação de Mestrado. Mestrado Profissional em Inovação Tecnológica e Propriedade Intelectual. Belo Horizonte: UFMG.

Chesnais, F., \&Sauviat, C. (2005). O Financiamento da Inovação no Regime Global de Acumulação Dominado pelo Capital Financeiro. In: Lastres, H. M. M., Cassiolato, J. E., \& Arroio, A. (2005). (eds.). Conhecimento, Sistemas de Inovação e de Desenvolvimento. Rio de Janeiro: Editora UFRJ e Contraponto.

Christensen, J. L. (1992). The role of Finance in National System of Innovation. In: Lundvall, B. (1992). National Systems of Innovation. London: Pinter Publishers.

Costa, A.A, Szapiro, M., \& Cassiolato, J.E. (2013). Análise da Operação do Instrumento de Subvenção Econômica à Inovação no Brasil. Conferência Internacional LALICS 2013 "Sistemas Nacionais de Inovação e Políticas de CTI para um Desenvolvimento Inclusivo e Sustentável", 11 e 12 de novembro- Rio de Janeiro, Brasil.

Dosi, et al. (1988). Technical Change and Economic Theory. London and New York: Pinter Publicher.

Fagerberg, J. (2005). Innovation: A Guide to the Literature. In: Fagerberg, J., Mowery, D.,\& Nelson, R. (2005). (eds.). The Oxford Handbook of Innovation. New York: Oxford University Press, 1-26.

Freeman, C., \& Soete, L. (2008). The Economics of Industrial Innovation. Cambridge, Massachusetts: The MIT Press.

Frenkel, J. (1993). Sistemas de apoio fiscal-crediticio ao risco tecnológico e à competitividade. In: Estudo da Competitividade da Indústria Brasileira (1993), Campinas: UNICAMP.

Guinet, J. (1995). National Systems for Financing Innovation, Paris: Head of Publications Service, OCDE.

Hall, B, H., \& Lerner, J. (2010). The Financing of R\&D and Innovation. In: Hall, B. H., \& Rosenberg, N.(2010). Handbook of the Economics of Innovation, Amsterdan: Elsevier.

Hall, B. (2002). The Financing of Research and Development. Oxford Review of Economic Polily, 18(1), 35-51.

Hasenclever, L., Paranhos, J., Chaves, G., \& Damasceno, C. (2016). Uma Análise das Políticas Industriais e Tecnológicas entre 2003-2014 e suas Implicações para o Complexo 
Industrial da Saúde. In: Hasenclever, L.,Paranhos, J., Chaves, G., \&Damasceno, C. (2016).Desafios de Operação e Desenvolvimento do Complexo Industrial da Saúde. Rio de Janeiro: E-papers, 99-126.

Interfarma. (2017). Guia Interfarma, São Paulo.Disponível em:https://www.interfarma.org.br/guia/guia-2017/apresentacao/.

Jiang, X., \& Wang, L. (2007). Process and Risk Analyses about Enterprises Technological Innovation. Chinese Business Review, 6(3), 49-54.

Knight, F.H. (1965). Risk, Uncertainty and Profits. London: Harper.

Koeller, P. (2009). Política Nacional de Inovação no Brasil: Releitura das Estratégias do Período 1995-2006. Tese de Doutorado de Doutorado da Universidade Federal do Rio de Janeiro.

Leonel, S. G. (2014). Mitos e Verdades sobre a Indústria de Venture Capital. Tese de Doutorado da Universidade Federal de Minas Gerais, Belo Horizonte/MG, 198 f. Disponível em <http://www.bibliotecadigital.ufmg.br/dspace/handle/1843/AMSA-9K9LVA>.

Longo, W. P., Krahe, P. R., \& Marinho, V.M.C. (2002). Incentivos Governamentais Não Fiscais: Estratégia para Fomentar a Inovação Tecnológica. Rio de Janeiro: FIRJAN, Cadernos de Tecnologia, 2.

Lundvall, B. Å., Johnson, B., Andersen, E.,\&Dalum, B.(2002). National Systems of Production, Innovation and Competence Building. Research Policy, 31, 213-231.

Mas-Colell, A., Whinston, M. D., \& Green, J.R. (1995). Microeconomic Theory. New York: Oxford University Press.

Mazzucato, M. (2013). Financing innovation: creative destruction vs. destructive creation. Industrial and Corporate Change, Oxford, v. 22, n. 4, julho, 2013, p. 851-867.

(2014). O Estado Empreendedor: Desmascarando o Mito do Setor Público vs Setor Privado. São Paulo: Portfólio-Penguin.

Montalban, M., \&Sakinç, M. E.(2013). Financialization and Productive Models in the Pharmaceutical Industry. Industrial and Corporate Change, Oxford, 22(4), 981-1030.

Nakamura, L. (1999). Intangibles: What put the New in the New Economy? Federal Reserve Bank of Philadelphia Business Review.

Nelson, R., \& Winter, S. (1982). An Evolutionary Theory of Economic Change. Cambridge: Havard University Press.

Nonaka, I., \&Takeuchi, H. (1997). Criação do conhecimento na empresa. Rio de Janeiro: Campus, $2^{\mathrm{a}}$ edição.

O’Sullivan, J. (2006). Finance and Innovation. In: Fagerberg, J., Mowery, D., \& Nelson, R. R. (2006). The Oxford Handbook of Innovation, Oxford University Press.

Paranhos, J. (2012). Interação entre Empresas e Instituições de Ciência e Tecnologia: O Caso do Sistema Farmacêutico de Inovação Brasileiro. Rio de Janeiro: Eduerj.

Paranhos, J., França, C., \& Mercadante, E. (2015). Sistemas Regionais de Inovação no Setor Farmacêutico Brasileiro: Estudo Comparativo entre o Rio de Janeiro e Estados 
Selecionados. In: Congresso Iberolatinoamericano de Gestão Tecnológica (ALTEC), Porto Alegre/RS, 19 a 22 de Outubro.

Paranhos, J., Mercadante, E., \& Hasenclever, L. (2020). Os Esforços Inovativos das Grandes Empresas Farmacêuticas no Brasil. Revista Brasileira de Inovação, 19, 1-28.

Paranhos, J., Perin, F., Vaz, M., Falcão, D., \& Hasenclever, L. (2020). Articulação de Políticas e Instrumentos de Produção e Inovação para o Complexo Industrial da Saúde no Brasil, 2003-2017: Os Casos do Inova Saúde e do Profarma. Relatório de Pesquisa Convênio UFRJ/IE/OSF.

Prochnik. V., Britto, J., Torres, R. L., \& Hasenclever, L. (2016). Inovação no Contexto de Convergência Tecnológica em Biotecnologia: Um Estudo de Caso. In: I Encontro Nacional da Economia Industrial e Inovação (ABEIN), Rio de Janeiro/RJ.

Roesch, S. M. A. (1999). Projetos de Estágio e de Pesquisa em Administração. São Paulo: Atlas, $2^{\mathrm{a}}$ edição.

Rosenberg, N. (1996). Uncertainty and Technological Change.Conference Series, Federal Reserve Bank of Boston.

Teixeira, A. L. S., Rapini, M. S., \& Szapiro, M. (2017). Discurso e Prática da Política Industrial no Período 2005-2014: Uma Avaliação da Distribuição Setorial dos Recursos Reembolsáveis do BNDES e da FINEP. In: II Encontro Nacional da Economia Industrial e Inovação (ABEIN), Rio de Janeiro/RJ.

Penedo,M. (2008). The Problem of Private under-investment in Innovation: A Policy Mind Map. Technovation, 28, 518-530.

Radaelli, V. (2006). A Inovação na Indústria Farmacêutica: Forças Centrípetas e Forças Centrífugas no Processo de Internacionalização. Dissertação de Mestrado da Universidade Estadual de Campinas (UNICAMP).

Schumpeter, J. (1911). The Theory of Economic Development, Cambridge: Harvard University Press.

Yin, R.K. (2001). Estudo de Caso: Planejamento e Método. Tradução de Daniel Grassi. Porto Alegre: Bookman, $2^{\text {a }}$ edição. 